\title{
EGFR NP_005219.2:p.A767_S768insTLA
}

National Cancer Institute

\section{Source}

National Cancer Institute. EGFR NP 005219.2:p.A767 S768insTLA. NCI Thesaurus. Code C98608.

An insertion of the amino acid sequence threonine-leucine-alanine between the alanine at position 767 and the serine at position 768 of the epidermal growth factor receptor protein. 